\title{
Different phenotypes of gastric fundic gland polyposis and cancer in patients with familial adenomatous polyposis depending on Helicobacter pylori infection
}

\author{
Yasuhiro Mitsui ${ }^{1} \cdot$ Ayaka Miyoshi $^{1} \cdot$ Koichi Okamoto $^{1} \cdot$ Naoki Muguruma $^{1} \cdot$ Jinsei Miyoshi $^{1} \cdot$ Kumiko Tanaka $^{1}$. \\ Shinji Kitamura ${ }^{1} \cdot$ Hiroshi Miyamoto $^{1}$ - Yasushi Sato ${ }^{1} \cdot$ Yoshimi Bando $^{2} \cdot$ Joji Shunto $^{3} \cdot$ Hidetaka Eguchi $^{4}$. \\ Yasushi Okazaki ${ }^{4} \cdot$ Hideyuki Ishida $^{5} \cdot$ Tetsuji Takayama $^{1}$ (D)
}

Received: 26 June 2019 / Accepted: 2 September 2019 / Published online: 16 September 2019

(c) The International Gastric Cancer Association and The Japanese Gastric Cancer Association 2019

\begin{abstract}
A 37-year-old male with tarry stool presented to our hospital. Esophagogastroduodenoscopy revealed advanced gastric cancer, fundic gland polyposis (FGPsis), and negativity for Helicobacter pylori (HP) infection. Computed tomography exhibited multiple liver tumors. Total colonoscopy (TCS) demonstrated 139 tubular adenomas. He was diagnosed as having unresectable gastric cancer and received systemic chemotherapy. His sister and mother had colorectal adenomatous polyposis as revealed by TCS. His sister had FGPsis and was negative for $H P$ infection, whereas his mother had early gastric cancer with $H P$ infection but not FGPsis. Genetic analysis revealed a novel mutation in exon 15 of the APC gene (NM_000038.5: c.7647_7648_delTG) for the patient, his mother, and his sister, whereas no mutation was found for his father who had no gastrointestinal polyps. Therefore, the pedigree was diagnosed as an FAP family with a novel APC germline mutation which had different gastric phenotypes depending on the status of $H P$ infection.
\end{abstract}

Keywords Familial adenomatous polyposis $\cdot A P C$ gene $\cdot$ Helicobacter pylori

Tetsuji Takayama

takayama@tokushima-u.ac.jp

1 Department of Gastroenterology and Oncology, Tokushima University Graduate School of Biomedical Sciences, 3-18-15, Kuramoto-cho, Tokushima city, Tokushima 770-8503, Japan

2 Division of Pathology, Tokushima University Hospital, 2-50-1, Kuramoto-cho, Tokushima city, Tokushima 770-8503, Japan

3 Shunto Clinic, 32-1, Matsushige-cho, Itano country, Tokushima 771-0220, Japan

4 Intractable Disease Research Center, Graduate School of Medicine, Juntendo University, Hongo 2-1-1, Bunkyo-ku, Tokyo 113-8421, Japan

5 Department of Digestive Tract and General Surgery, Saitama Medical Center, Saitama Medical University, 1981 Kamoda-Tsujido-cho, Kawagoe city, Saitama 350-8550, Japan

\section{Introduction}

Familial adenomatous polyposis (FAP) is an autosomal dominant disease characterized by multiple colonic adenomas caused by a germline mutation of the adenomatous polyposis coli $(A P C)$ gene [1-4]. FAP patients exhibit various upper gastrointestinal manifestations; namely, gastric adenoma/cancer and fundic gland polyposis (FGPsis) of the stomach [5]. In general, sporadic fundic gland polyps (FGPs) have been considered to be benign lesions because of its little or no malignant potential $[6,7]$. Moreover, although FGPsis has also been regarded as a benign lesion, in 1999, Hofgärtner and colleagues reported a case of an FAP patient who had gastric cancer related with FGPs [8], and since then FGPsis has been regarded as a precursor lesion of carcinoma.

Helicobacter pylori $(H P)$ is a principal cause of gastric cancer via atrophic gastritis [9]. Although a correlation between the number of FGPs in FAP patients and HP infection has been reported [10], the clinicopathological features of gastric cancers and FGPsis and the involvement of $H P$ infection in carcinogenesis in FAP patients have not yet been 
clarified. Herein, we report an FAP pedigree with a novel $A P C$ germline mutation who showed different phenotypes of FGPsis and gastric cancer depending on $H P$ infection status.

\section{Case report}

A 37-year-old Japanese male with tarry stool presented for further investigation. At age 18, he had FGPsis revealed by esophagogastroduodenoscopy (EGD), but no other past medical history was identified and no antacid drugs had been administered. His grandfather had died from gastric cancer; however, it was unclear whether or not he had gastrointestinal polyposis. Laboratory data demonstrated anemia (Hb $12.3 \mathrm{~g} / \mathrm{dL}$ ), elevation of tumor marker (CA19-9 1603 $\mathrm{U} / \mathrm{mL}$ ), and seronegativity for $H P$ measured by an enzyme immunoassay. EGD indicated profuse FGPsis (Fig. 1a) and advanced gastric cancer in the gastric fundus (Fig. 1b), but no polyposis in the antrum or duodenum. A biopsy specimen obtained from the fundus tumor indicated papillary adenocarcinoma (Fig. 1c), and typical FGPs originating from profuse gastric polyps (Fig. 1d). Colonoscopy revealed 139 colorectal polyps, and some biopsy specimens showed typical pathological findings of tubular adenomas (Fig. 1e, f ).
Computed tomography indicated multiple liver metastases and regional lymph node swelling. Therefore, he was diagnosed with unresectable gastric cancer cT3N1M1 at stage IV according to the Japanese classification of gastric carcinoma [11]. He had received systemic chemotherapy, i.e., docetaxel, cisplatin, and S-1 combined therapy, followed by weekly paclitaxel therapy and irinotecan therapy; however, his disease progressed and he finally died 14 months after diagnosis.

His family received colonoscopy based on suspicion of FAP. His mother and sister had colorectal adenomatous polyposis (about 100 polyps each) but his father did not. Thus, this pedigree was diagnosed as FAP. Figure 2 shows the patient's family pedigree and their gastrointestinal phenotypes. His sister had FGPsis (Fig. 3a), but his father (Fig. 3b) and mother (Fig. 3c) did not. In addition, his mother had been infected with $H P$ and had early gastric cancer in the gastric body (Fig. 3d). She underwent endoscopic submucosal dissection for the lesion. A pathological examination revealed complete resection of welldifferentiated tubular adenocarcinoma (Fig. 3e, f). She was then successfully treated by eradication therapy for $H P$ using amoxicillin, clarithromycin, and lansoprazole. However, it is important to note that when she underwent

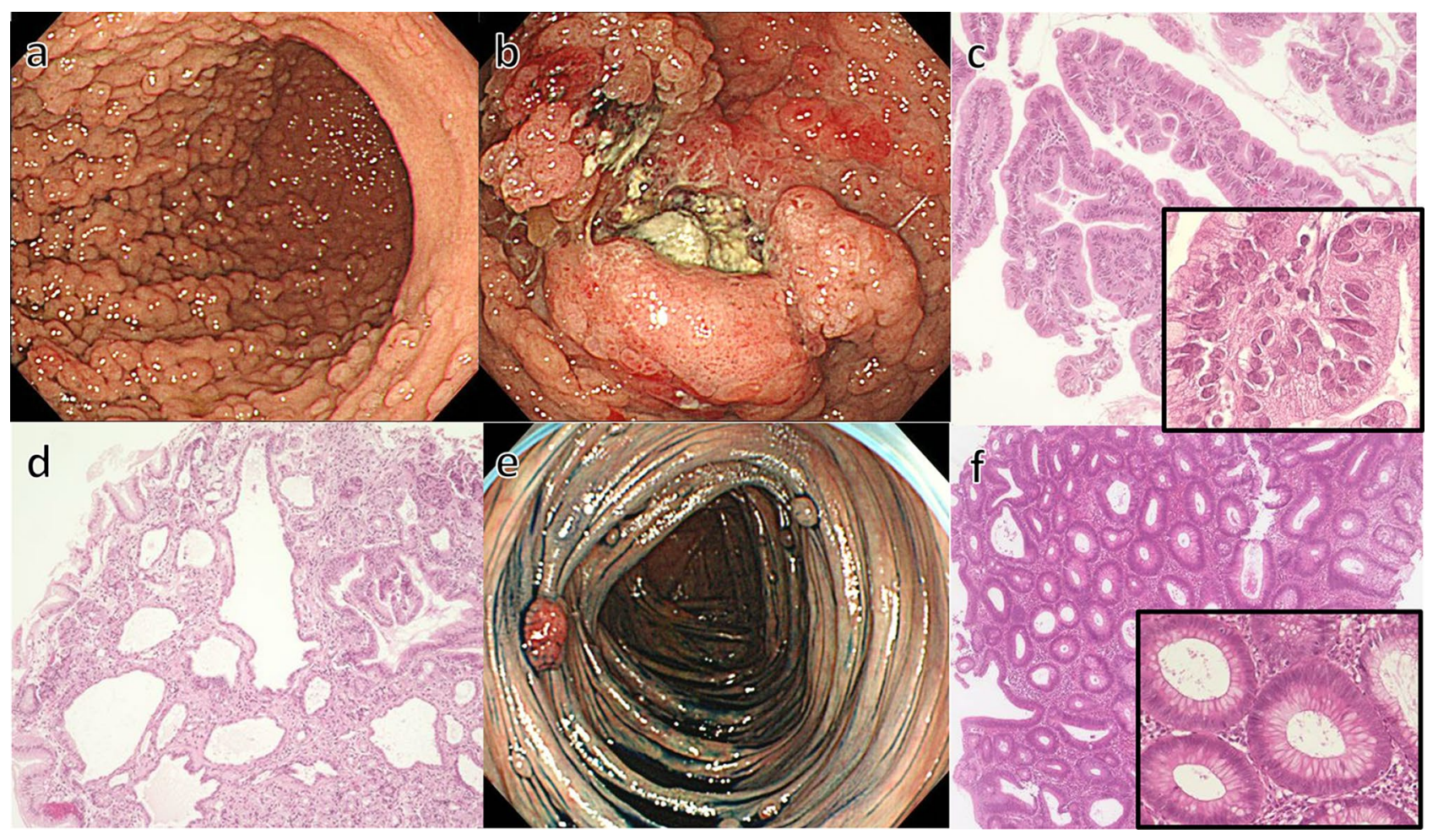

Fig. 1 Esophagogastroduodenoscopy (EGD) image at the first medical examination and pathological findings of biopsy specimen. a Profuse fundic gland polyposis in the fundic gland area. b Adenocarcinoma in the gastric fundus classified as Type 2. c A papillary adenocarcinoma (pap). d Fundic gland polyp with cystically dilated gland. e, f Multiple colonic adenoma stained with indigo carmine and its typical pathological findings 


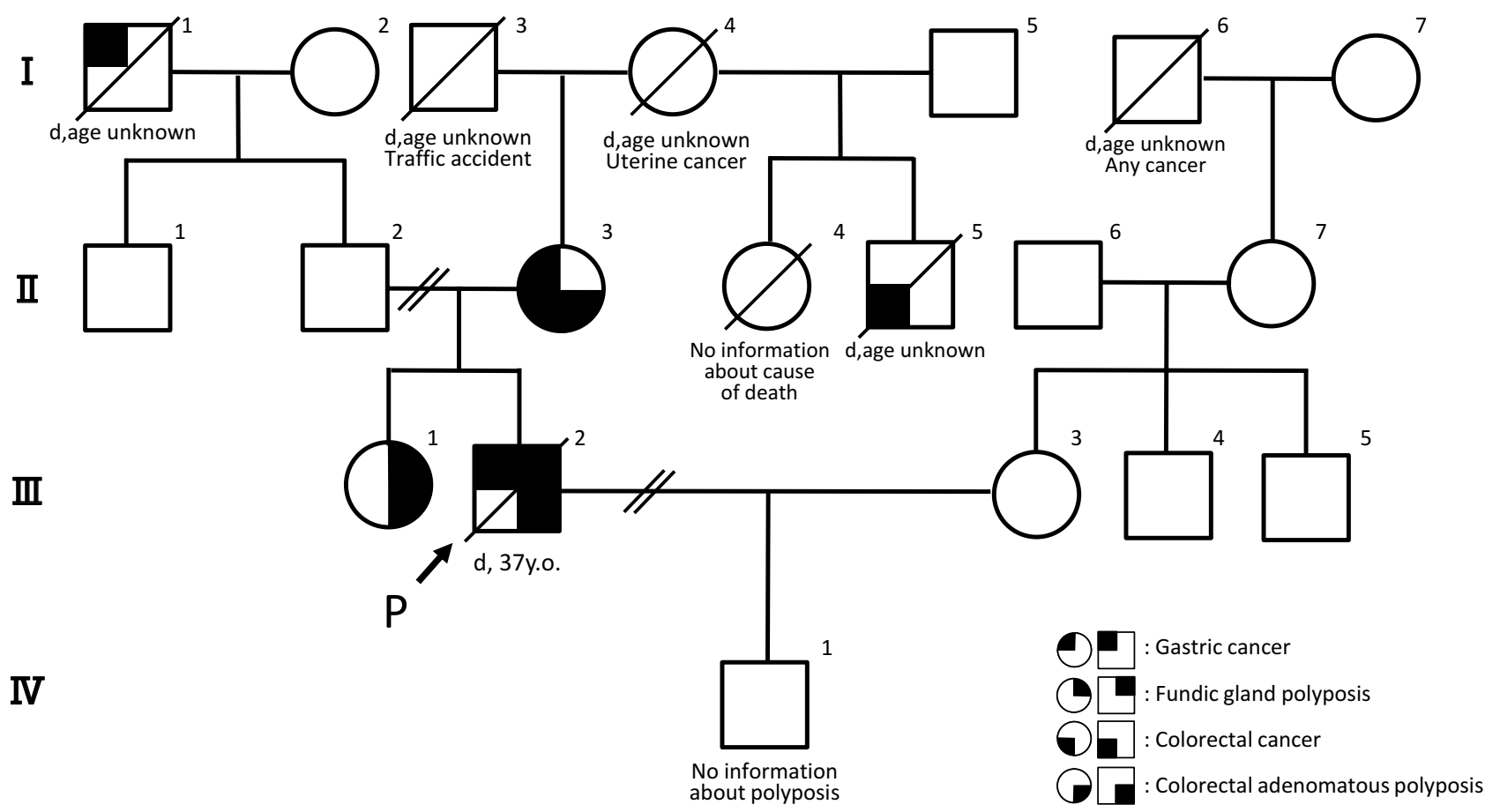

Fig. 2 Pedigree of the family

EGD 6 years after the eradication of $H P$, multiple FGPs were identified in her gastric fundus (Fig. $3 \mathrm{~g}$ ). A biopsy of the polyps exhibited typical pathological findings of FGPs (Fig. 3h, i). Thus, the gastric phenotype of the mother seemingly changed from the initial phenotype after eradication of $H P$.

Since it was assumed that the pedigree had germline variants, we performed a genetic analysis of the $A P C$ gene from genomic DNA of peripheral blood of the family members using a polymerase chain reaction directsequencing method, after obtaining informed consent. A novel frameshift mutation, NM_000038.5: c.7647_7648_ delTG (p.Glu2550Alafs*32), was found in exon 15 of the $A P C$ gene in the patient (Fig. 4a), his sister (Fig. 4b), and his mother (Fig. 4c), but not in his father (Fig. 4d).

Histopathological examination with immunostaining showed that adenocarcinoma of the proband was positive for MUC5AC but negative for MUC2 and CDX2 (Fig. 5a, $\mathrm{b}, \mathrm{c})$, suggesting a gastric foveolar type, while his mother's adenocarcinoma was appeared to be the intestinal type because of accumulation of CDX2 in the nuclei of cancer cells (Fig. 5f, g, h). Moreover, accumulation of p53 protein was detected in the nuclei of both adenocarcinomas (Fig. 5 d, i), whereas $\beta$-catenin was positive for the proband but negative for his mother (Fig. 5e, f, j).

\section{Discussion}

FAP patients often have upper gastrointestinal tumors. FGPsis is the most common gastric manifestation in FAP patients, with incidence ranging from 20 to $88 \%$. Abraham and associates demonstrated that FAP-related FGPs sometimes show foveolar dysplasia and more frequently exhibit somatic mutation of the $A P C$ gene compared with sporadic FGPs [6]. In Western countries, gastric cancer occurs at a roughly similar rate among FAP and non-FAP patients [12], whereas an increased risk of gastric cancer in Asian FAP patients has been reported [13]. Among Japanese FAP patients, the incidence of gastric cancer has been reported to range from 4.5 to $13.6 \%$ [14, 15], and the relative mortality risks were 2.7 for male and 4.2 for female patients [12]. The mortality rate due to gastric cancer among FAP patients is $2.8 \%$ [16], and the risk is heightened for patients who have undergone prophylactic colectomies [17].

The average age of onset for FAP-related gastric cancer was reportedly $49.2 \pm 12.7$ years and no significant difference between males and females was found [12]. FAP-related proximal gastric cancer often arise in a background of FGPs that coalesce [18]. In the present case, 

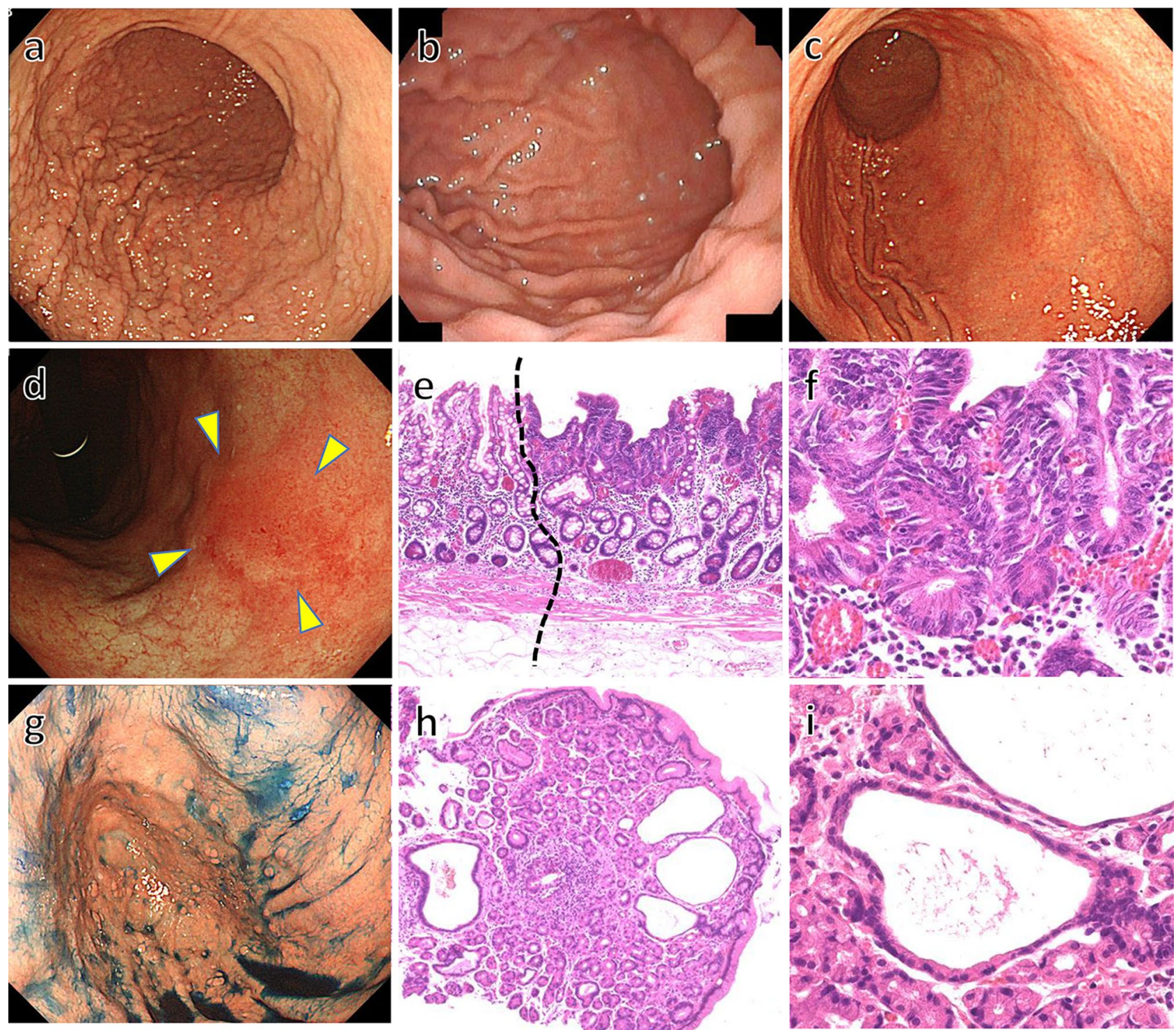

Fig. 3 EGD and pathological findings from related family members of the proband. a Fundic gland polyposis in his sister. b No polyposis in his father. c No polyposis but atrophic gastritis (opened type according to Kimura-Takemoto classification) existed in his mother at initial examination. d Early gastric cancer in the gastric body of his mother. e, f Pathological findings of the cancer removed by endo-

the proband at a relatively young age had proximal gastric cancer in a background of FGPsis, consistent with typical FAP-related gastric cancer. In contrast, his mother simultaneously had well-differentiated gastric cancer in the atrophic mucosa, consistent with $H P$-induced gastric cancer. The survival time of the proband was 14 months despite ample systemic chemotherapy, which was similar to sporadic cases [19]. However, rapid growth of metastatic sites suggested that unresectable FAP-related gastric cancer might have greater malignant potential as compared with sporadic cases. scopic submucosal dissection (0-IIa, tub1, M, according to Japanese Classification of Gastric Carcinoma). g EGD image of his mother 6 years later after eradication of Helicobacter pylori. Fundic gland polyps were observed. h, i Typical pathological findings of fundic gland polyp

Germline mutations in the $A P C$ gene are detectable in a majority of FAP patients [20, 21]. These germline variants of the $A P C$ gene are mostly frameshift or nonsense mutations, leading to a truncated $A P C$ protein, consequent nuclear accumulation of $\beta$-catenin, and finally, enhancement of cell proliferation. Additional mutations of the KRAS or $P 53$ genes have been reported to cause carcinogenesis [22, 23], suggesting that the adenoma-carcinoma sequence plays an important role in gastric cancer pathogenesis in FAP patients [24]. Immunohistochemical findings in the proband were consistent with the adenoma-carcinoma sequence; i.e., 
a
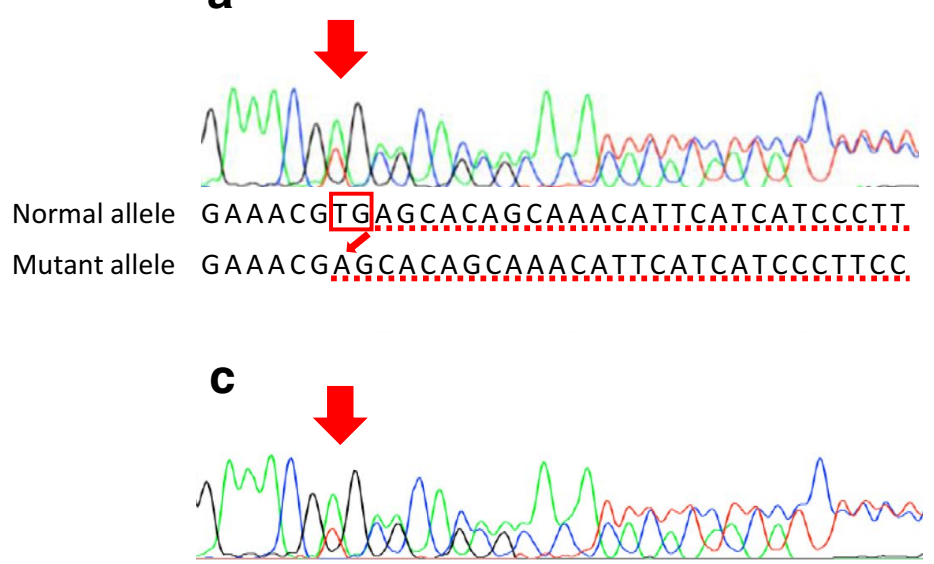

Normal allele GAAACG TGAGCACAGCAAACATTCATCATCCCTT Mutant allele GAAACGAGCACAGCAAACATTCATCATCCCTTCC b
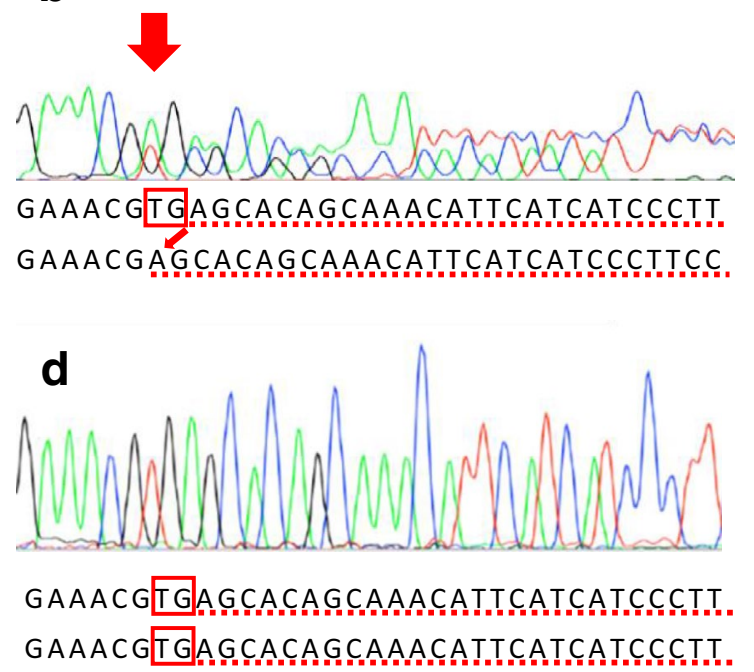

Fig. 4 An APC exon15 germline mutation in the patient and his family. A novel frameshift mutation, c.7647_7648_delTG, was detected, as revealed by polymerase chain reaction direct sequencing. a The proband, $\mathbf{b}$ his sister, $\mathbf{c}$ his mother, $\mathbf{d}$ his father

accumulation of p53 and $\beta$-catenin in the nuclei of cancer cells. However, other oncogenesis pathways also might exist given the negativity for $\beta$-catenin of cancer cells in his mother, which represented "sporadic carcinogenesis" of intestinal-type gastric cancer.

Correlations between the APC gene mutation site and FAP phenotype have been reported. FAP patients with germline mutation at the $3^{\prime}$ distal end of the $A P C$ gene could have attenuated colonic polyposis and upper gastrointestinal tumors [25]. In this pedigree, although the family members with colonic adenomatous polyposis had the same germline mutation as the proband at the $3^{\prime}$ distal end of the APC gene, a healthy individual did not, suggesting that this mutation is responsible for their phenotype. However, the gastric phenotype of the proband's mother affected by $H P$ was different from other members with the same $A P C$ mutation. Nakamura and coworkers reported that the prevalence of FGPs in FAP patients affected by $H P$ was significantly lower than that of patients without $H P$ [10]. Although the precise mechanism for involution of FGPs is unclear, $H P$ infection could alter the direction of the differentiation process of fundic gland progenitor cells from a glandular cell lineage towards a pit cell lineage [26]. Thus, the FGPs might regress in the mother due to $H P$ infection, consistent with relapse of FGPs following $H P$ eradication. Conversely, $H P$ injects CagA protein into epithelial cells, and CagA is reportedly associated with high positivity for $p 53$ mutation [27]. Therefore, it is possible that gastric cancer of the intestinal type might develop in the background mucosa with chronic gastritis and intestinal metaplasia as a result of $p 53$ mutation occurring before $\beta$-catenin accumulation. Since $H P$ infection is more common among Asian people, including the Japanese population [28], careful assessment is necessary for FAP patients, in view of the phenotypic change associated with $H P$ infection.

Despite the improvement of survival in FAP patients with appropriate treatment and surveillance for colonic polyposis, management of FAP-related gastric neoplasm remains insufficient. Henceforth, attention should be paid to FGPsis due to the heightened malignant potential. Additionally, it is particularly important to distinguish familial FGPs from sporadic cases, paying attention to the status of $H P$ infection. 


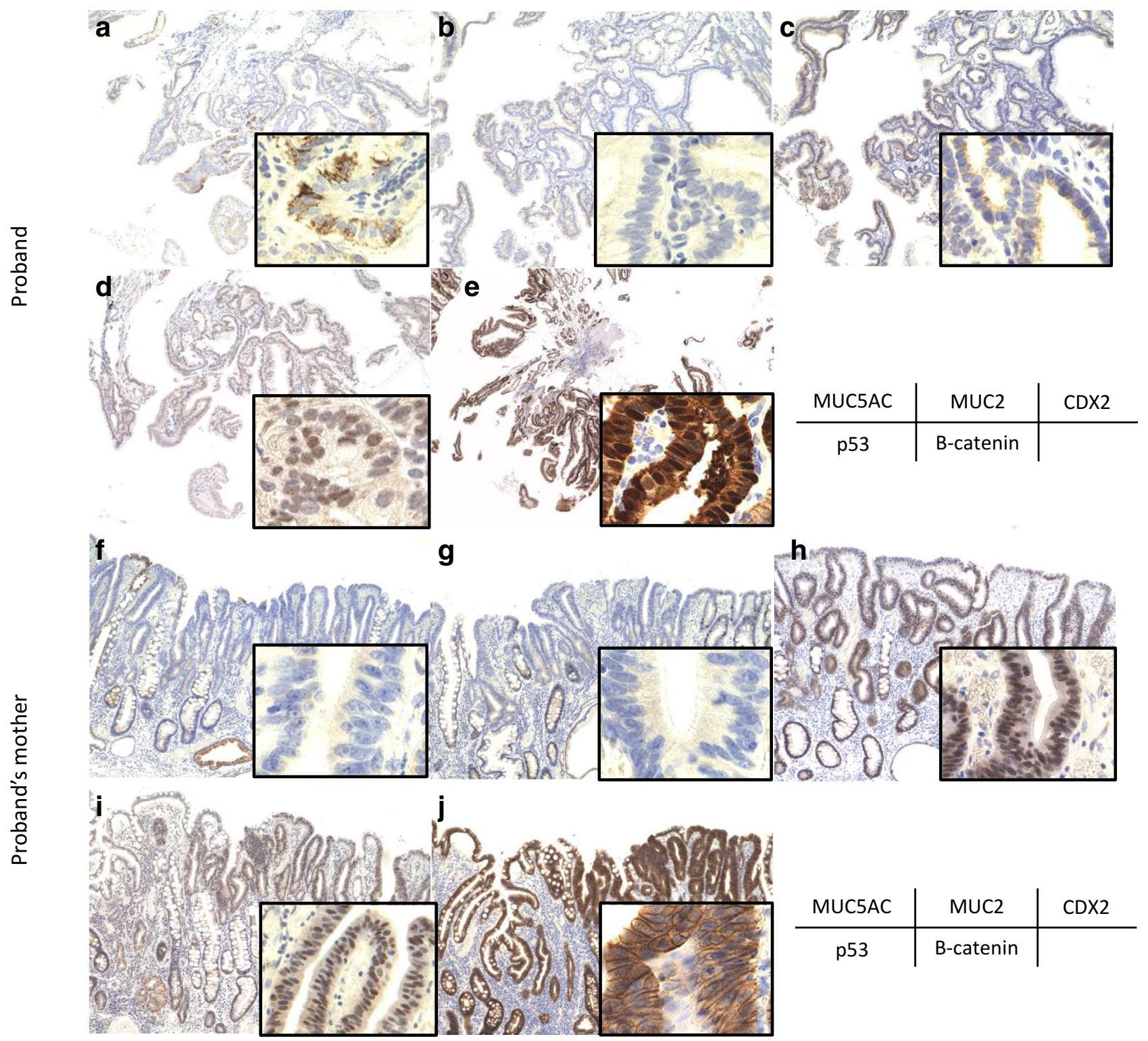

Fig. 5 Immunohistochemical findings in adenocarcinoma of the proband (a-e) and his mother $(\mathbf{f}-\mathbf{j})$. a, b, $\mathbf{c}$ Positive for MUC5AC but negative for MUC2 and CDX2 (foveolar type). d, e Positively stained for $\beta$-catenin and $\mathrm{p} 53$ in nuclei. $\mathbf{f}, \mathbf{g}, \mathbf{h}$ Positive for CDX2 but negative for MUC2 and MUC5AC. i, j Positively stained for p53 in nuclei but negative for $\beta$-catenin (membranous pattern)

\section{Compliance with ethical standards}

Conflict of interest The authors declare that they have no conflict of interest.

Ethical approval All patients participated in the SGHGCS trial, which was conducted with the approval of the local ethics committee of Saitama Medical Center (No. 924) and Saitama Medical University (No.747) in accordance with the Helsinki Declaration. Informed consent was obtained from all patients.

\section{References}

1. Gorden J, Thliveris A, Samowitz W, Carlson M, Albertsen H, et al. Identification and characterization of the adenomatous polyposis coli gene. Cell. 1991;66:589-600.

2. Joslyn G, Carlson M, Thliveris A, Albertsen H, Gelbert L, et al. Identification of deletion mutations and three new genes at the familial polyposis locus. Cell. 1991;66:601-13.

3. Kinzler KW, Nilbert MC, Su LK, Vogelstein B, Bryan TM, et al. Identification of FAP locus genes from chromosome 5q21. Science. 1991;253:661-5.

4. Nishisho I, Nakamura Y, Miyoshi Y, Miki Y, Ando H, et al. Mutations of chromosome $5 \mathrm{q} 21$ genes in FAP and colorectal cancer patients. Science. 1991;253:665-9. 
5. Yamaguchi T, Ishida H, Ueno H, Kobayashi H, Hinoi T, et al. Upper gastrointestinal tumours in Japanese familial adenomatous polyposis patients. Jpn J Clin Oncol. 2016;46:310-5.

6. Abraham SC, Nobukawa B, Giardiello FM, Hamilton RS, Wu TT. Fundic gland polyps in familial adenomatous polyposis: neoplasm with frequent somatic adenomatous polyposis coli gene alterations. Am J Pathol. 2000;157:747-54.

7. Wu TT, Kornacki S, Rashid A, Yardley JH, Hamilton SR. Dysplasia and dysregulation of proliferation in foveolar and surface epithelia of fundic gland polyps from patients with familial adenomatous polyposis. Am J Surg Pathol. 1998;22:293-8.

8. Hofgärtner WT, Thorp M, Ramus MW, Delorefice G, Chey WY, Ryan CK, et al. Gastric adenocarcinoma associated with fundic gland polyps in a patient with attenuated familial adenomatous polyposis. Am J Gastroenterol. 1999;94:2275-81.

9. Huang JQ, Sridhar S, Chen Y, Hunt RH. Meta-analysis of the relationship between Helicobacter pylori seropositivity and gastric cancer. Gastroenterology. 1998;114:1169-79.

10. Nakamura S, Matsumoto T, Kobori Y, Iida M. Impact of Helicobacter pylori infection and mucosal atrophy on gastric lesions in patients with familial adenomatous polyposis. Gut. 2002;51:485-9.

11. Japanese Gastric Cancer Association. Japanese classification of gastric carcinoma: 3rd English edition. Gastric Cancer. 2011;14:101-12.

12. Offerhaus GJ, Giardiello FM, Krush AJ, Booker SV, Termette $\mathrm{AC}$, et al. The risk of upper gastrointestinal cancer in familial adenomatous polyposis. Gastroenterology. 1992;102:1980-2.

13. Iwama T, Mishima Y, Utsunomiya J. The impact of familial adenomatous polyposis on the tumorigenesis and mortality at the several organs. Its rational treatment. Ann Surg. 1993;217:101-8.

14. Itoh $\mathrm{H}$, Ikeda $\mathrm{S}$, Oohata $\mathrm{Y}$, Iida $\mathrm{M}$, Inoue $\mathrm{T}$, et al. Treatment of desmoid tumors in Gardner's syndrome. Report of a case. Dis Colon Rectum. 1988;31:459-61.

15. Watanabe H, Enjoji M, Yao T, Ohsato K. Gastric lesions in familial adenomatosis coli: their incidence and histologic analysis. Hum Pathol. 1978;9:269-83.

16. Iwama T, Tamura K, Morita T, Hirai T, Hasegawa H, et al. A clinical overview of familial adenomatous polyposis derived from the database of the Polyposis Registry of Japan. Int J Clin Oncol. 2004;9:308-16.

17. Offerhaus GJ, Entius MM, Giardiello FM. Upper gastrointestinal polyps in familial adenomatous polyposis. Hepatogastroenterology. 1999;46:667-9.
18. Leone PJ, Mankaney G, Sarvapelli S, Abushamma S, Lopez R, et al. Endoscopic and histologic features associated with gastric cancer in familial adenomatous polyposis. Gastrointest Endosc. 2019;89:961-8.

19. Koizumi W, Narahara H, Hara T, Takagane A, Akiya T, Takagi M, et al. S-1 plus cisplatin versus S-1 alone for first-line treatment of advanced gastric cancer (SPIRITS trial): a phase III trial. Lancet Oncol. 2008;9:215-21.

20. Groden J, Thliveris A, Samowitz W, Carison M, Gelbert L, Albertsen $\mathrm{H}$, et al. Identification and characterization of the familial adenomatous polyposis coli gene. Cell. 1991;66:589-600.

21. Miyoshi Y, Nagase H, Ando H, Horii A, Ichii S, Nakatsuru S, et al. Somatic mutations of the APC gene in colorectal tumors: mutation cluster region in the APC gene. Hum Mol Genet. 1992;1:229-33.

22. Takayama T, Ohi M, Hayashi T, Miyanishi K, Nobuoka A, et al. Analysis of K-ras, APC, and beta-catenin in aberrant crypt foci in sporadic adenoma, cancer, and familial adenomatous polyposis. Gastroenterology. 2001;121:599-611.

23. Will OC, Leedha SJ, Elia G, Phillips RK, Clark SK, et al. Location in the large bowel influence the APC mutations observed in FAP adenomas. Fam Cancer. 2010;9:389-93.

24. Vogelstein B, Papadopoulos N, Velculescu VE, Zhou S, Diaz LA Jr, et al. Cancer genome landscapes. Science. 2013;339:1546-58.

25. Nieuwenhuis MH, Vasen HF. Correlations between mutation site in APC and phenotype of familial adenomatous polyposis (FAP): a review of the literature. Crit Rev Oncol Hematol. 2007;61:153-61.

26. Watanabe N, Seno H, Nakajima T, Yazumi S, Miyamoto S, et al. Regression of fundic gland polyps following acquisition of Helicobacter pylori. Gut. 2002;51:742-5.

27. Shibata A, Parsonnet J, Longacre TA, Garcia MI, Puligandla $\mathrm{B}$, et al. CagA status of Helicobacter pylori infection and p53 gene mutations in gastric adenocarcinoma. Carcinogenesis. 2002;23:419-24.

28. Hunt RH, Xiao SD, Megraud F, Leon-Barua R, Bazzoli F, van der Merwe S, et al. Helicobacter pylori in developing countries. World gastroenterology organization global guideline. J Gastrointestin Liver Dis. 2011;20:299-304.

Publisher's Note Springer Nature remains neutral with regard to jurisdictional claims in published maps and institutional affiliations. 